\section{OP46 \\ NOVEL RISK FACTORS FOR MENORRHAGIA AND DYSMENORRHEA IN ADOLESCENCE USING THE ALSPAC COHORT}

1,2Florence Z Martin* 1,2 Kayleigh E Easey 1,2 Laura D Howe, 1,2,3 Deborah A Lawlor ${ }^{1,2,3}$ Abigail Fraser, ${ }^{1,2}$ Caroline L Relton, ${ }^{1,2}$ Gemma C Sharp. ${ }^{1} M R C$ Integrative Epidemiology Unit, University of Bristol, Bristol, UK; ${ }^{2}$ Department of Population Health Sciences, University of Bristol, Bristol, UK; ${ }^{3}$ NIHR Bristol Biomedical Research Centre, University of Bristol, Bristol, UK

\subsection{6/jech-2021-SSMabstracts.46}

Background Precise estimates of prevalence vary, but menstrual symptoms such as menorrhagia (heavy or prolonged bleeding) and dysmenorrhea (pain associated with period) are experienced by a large proportion of adolescent girls. Risk factors, co-morbidities and potential impacts of these menstrual problems on other areas of health and wellbeing are not well-characterised. We aimed to describe the prevalence of menorrhagia and dysmenorrhea in the Avon Longitudinal Study of Parents and Children (ALSPAC) cohort and to identify associations between these symptoms and other traits.

Methods Cases of both dysmenorrhea and menorrhagia were identified using self-report questionnaires administered nine times between the ages of 8 and 17 years ( $n=4,222$ responded to at least one). Cases were defined as respondents who had reported to have visited the doctor for the symptom, any time during puberty. We used the ALSPAC cohort's corresponding epigenetic data resource, ARIES, to identify differences in methylation between cases for each symptom and those who never had reported it (controls), to identify traits associated with each symptom. These identified traits were then explored in the full ALSPAC cohort, using logistic regression.

Results Of the 4,222 adolescents who had responded to at least one of the puberty questionnaires, almost 70\% ( $\mathrm{n}=$ 2,915 ) had experienced dysmenorrhea at least once during puberty and over 50\% ( $\mathrm{n}=2,123)$ had experienced menorrhagia. Of these, 22\% $(\mathrm{n}=641)$ and $25 \%(\mathrm{n}=527)$ visited the doctor for dysmenorrhea and menorrhagia, respectively. These symptoms showed significant overlap with one another, but remained distinctive. The epigenetic findings revealed potential associations with novel traits, including inflammatory markers and child abuse. In ALSPAC, both symptoms were shown to be associated with increased Creactive protein and higher average adverse childhood experience (ACE) score, among other traits including prenatal smoke exposure, higher average body mass index and lower socioeconomic position.

Discussion The prevalence of both dysmenorrhea and menorrhagia is high in ALSPAC, highlighting an important and neglected area for population health research and intervention. These findings may suggest that exposure to ACE may go on to increase likelihood of enduring more severe menstrual symptoms in adolescence, potentially mediated by higher BMI and circulating inflammatory proteins. The implication of ACE adds to the growing body of evidence that they negatively affect long-term health. This study overall describes novel associations between menstrual symptoms and early life exposures that warrant further investigation.

\section{OP47 ENDOGENOUS HORMONES AND RISK OF INVASIVE BREAST CANCER IN PRE- AND POST-MENOPAUSAL WOMEN: FINDINGS FROM THE UK BIOBANK}

Sandar Tin Tin*, Gillian K Reeves, TImothy J Key. Cancer Epidemiology Unit, The University of Oxford, Oxford, UK

\subsection{6/jech-2021-SSMabstracts.47}

Background Circulating sex hormones and growth factors have been associated with the risk of invasive breast cancer, but the nature of these relationships is not fully understood. We used data from UK Biobank, a large study with hormone measures on the full cohort and repeat measures in a sub-sample, to estimate the magnitudes of the associations after allowing for measurement error.

Methods We included 30,565 pre-menopausal and 133,294 post-menopausal women in this analysis. Hormone concentrations were measured in serum collected between 2006 and 2010, and incident cancer cases were identified through linkage to national cancer and death registries. Multivariable Cox proportional hazards models were used, and hazard ratios (HRs) were corrected for regression dilution using repeat measures collected in about 5,000 women four years after recruitment (except for oestradiol).

Results During a median follow-up of 7.1 years, 527 pre-menopausal and 2,997 post-menopausal women were diagnosed with invasive breast cancer. Cancer risk was positively associated with testosterone in post-menopausal women (HR per $0.5 \mathrm{nmol} / \mathrm{L}$ increment: $1.18 ; 95 \% \mathrm{CI}: 1.14,1.23)$ but not in premenopausal women ( $p_{\text {heterogeneity }}=0.03$ ), and with IGF-1 (insulin-like growth factor-1) (HR per $5 \mathrm{nmol} / \mathrm{L}$ increment: $1.18 ; 1.02,1.35$ (pre-menopausal) and $1.07 ; 1.01,1.12$ (postmenopausal); $p_{\text {heterogeneity }}=0.2$ ), and inversely associated with SHBG (sex hormone binding globulin) (HR per $30 \mathrm{nmol} / \mathrm{L}$ increment: $0.96 ; 0.79,1.15$ (pre-menopausal) and $0.89 ; 0.84$, 0.94 (post-menopausal); $p_{\text {heterogeneity }}=0.4$ ). Oestradiol, assessed only in pre-menopausal women, was not associated with risk, but there were study limitations for this hormone.

Conclusion This study confirms associations of testosterone, IGF-1 and SHBG with breast cancer risk, with heterogeneity by menopausal status for testosterone.

\section{OP48 IMPLEMENTATION OF HIP FRACTURE SERVICES: A QUALITATIVE STUDY USING EXTENDED NORMALIZATION PROCESS THEORY}

Sarah Drew*, Fiona Fox, Celia Gregson, Rachael Gooberman-Hill. Musculoskeletal Research Unit, Translational Health Sciences, University of Bristol, Bristol, UK

\subsection{6/jech-2021-SSMabstracts.48}

Background Hip fractures are a devastating injury with high healthcare costs. Despite national standards and guidelines, there is substantial variation in hospital delivery of hip fracture care and in patient outcomes. This study aimed to understand organisational processes that help or hinder the implementation of hip fracture services, using extended Normalization Process Theory (eNPT), which specifies four constructs that impact on successful implementation: capacity, potential, capability and contribution. 